\title{
A new method on diagnostics of muons produced by a short pulse laser
}

\author{
Feng Zhang, Boyuan Li, Lianqiang Shan, Bo Zhang, Wei Hong, and Yuqiu Gu \\ Science and Technology on Plasma Physics Laboratory, Research Center of Laser Fusion, CAEP, Mianyang 621900, China \\ (Received 16 November 2016; revised 14 April 2017; accepted 5 May 2017)
}

\begin{abstract}
Muons produced by a short pulse laser can serve as a new type of muon source having potential advantages of high intensity, small source emittance, short pulse duration and low cost. To validate it in experiments, a suitable muon diagnostics system is needed since high muon flux generated by a short pulse laser shot is always accompanied by high radiation background, which is quite different from cases in general muon researches. A detection system is proposed to distinguish muon signals from radiation background by measuring the muon lifetime. It is based on the scintillator detector with water and lead shields, in which water is used to adjust energies of muons stopped in the scintillator and lead to against radiation background. A Geant 4 simulation on the performance of the detection system shows that efficiency up to $52 \%$ could be arrived for low-energy muons around $200 \mathrm{MeV}$ and this efficiency decreases to $14 \%$ for high-energy muons above $1000 \mathrm{MeV}$. The simulation also shows that the muon lifetime can be derived properly by measuring attenuation of the scintilla light of electrons from muon decays inside the scintillator detector.
\end{abstract}

Keywords: diagnostics; Geant4 simulation; muon source; short pulse laser

\section{Introduction}

A muon ${ }^{[1,2]}$ is one of the elementary particles in fundamental physics which belongs to the second generation of leptons. It has a spin of $1 / 2$ and a mass of $105.7 \mathrm{MeV} / \mathrm{c}^{2}$ intermediate between the proton and the electron. Since the discovery in the cosmic rays research in 1936, muon was a popular research object in particle physics and applied physics. Due to its larger mass compared to that of electron, a muon does not produce significant synchrotron radiation, consequently negligible bremsstrahlung which is at an advantage of muon collider and related neutrino physics ${ }^{[3,4]}$. Polarized muon source can also be applied as a suitable probe in many disciplines such as material science, biomedical, superconductor physics and so on ${ }^{[5]}$. Benefitting from its high penetrability, a muon can go through more than hundreds of $\mathrm{g} / \mathrm{cm}^{2}$ which is much longer than X-ray radiography ${ }^{[6]}$. Therefore, muon radiography can be applied on imaging of dense object such as nuclear materials ${ }^{[7-9]}$.

In general, the muon is produced as a secondary cosmic ray from the $\pi$ and $\mathrm{K}$ meson decays by the interactions of the primary cosmic ray protons with nuclei (N, O atoms) in the air ${ }^{[2]}$. The intensity of cosmic muon flux is $1 \mathrm{~cm}^{-2} \cdot \mathrm{min}^{-1}$ with a mean energy at $4 \mathrm{GeV}$. Hence, cosmic ray muon

Correspondence to: Y. Gu, Academy of Engineering Physics, Mianyang 621900, China. Email: yqgu@ caep.ac.cn source has low intensity, high energy, and a very long stopping range. Muons can also be produced by accelerators through the decay of mesons which are produced in nuclear interaction between accelerated protons and nuclear targets. The accelerator muon source has high intensity and a controllable stopping range for the energy covering from $\mathrm{eV}$ to hundreds of MeV. However, high budget makes it impossible to be applied commonly in laboratories except finite facilities in the world ${ }^{[10-13]}$.

Another possible source of a muon with both signs is the Bethe-Heitler lepton pair production process ${ }^{[1,14-16]}$

$$
\gamma+A \rightarrow A^{\prime}+\mu^{+} \mu^{-}
$$

in high-energy photon interactions with high $\mathrm{Z}$ materials. Although the muon pair production in this process is suppressed relative to the electron-positron pair production by a factor of $\left(m_{e} / m_{\mu}\right)^{2} \approx 10^{-4}$, a muon source can still be realized this way ${ }^{[17,18]}$. In fact, along with the development of petawatt laser in the last decade, electrons have been produced and accelerated to several $\mathrm{GeV}$ which have exceeded the threshold of muon generation by the laser wakefield acceleration method ${ }^{[19-22]}$. The typical electron flux is at the magnitude of tens of $\mathrm{pC}$ corresponding to $\sim 10^{8}$ electrons in a bunch, and would produce $1 \times 10^{2}$ muon pairs with pair energy centered around $1 \mathrm{GeV}$ as expected by Titov 
in Ref. [23]. An even higher production of $10^{6}$ dimuons was also promising based on a $100 \mathrm{~J}$ petawatt laser facility. This type of muon source would have the advantage of high intensity, small source emittance, short pulse duration and low cost. It can be used in fundamental problems of elementary physics such as neutrino physics, lepton physics and can also be used in muon spin relaxation ( $\mu \mathrm{SR})$, muon radiography and so on.

To validate the dimuon production by a short pulse laser, a suitable muon diagnostics is needed. Although there were lots of muon detectors since its discovery ${ }^{[24-27]}$, such as Cerenkov Detector, Drift Chamber, Time Projection Chamber, Multiwire Proportional Chamber, Resistive Plate Chamber and so on, all of the detectors are designed for a single muon event, not suitable for high-intensity measurement. Besides, these kinds of detectors generally need a complex magnet system, gas system and electronics readout system, and are thus very expensive. On the other hand, since laser muons were mainly generated by the high-energy photons from bremsstrahlung radiation of laser wakefield accelerated electrons, a strong radiation background was generated simultaneously such as photons, electrons, protons and secondary neutrons which would highly saturate the detectors. How to distinguish the muon signal from the strong radiation background is a key question in the laserbased muon source.

Our idea is to diagnose the muon lifetime to distinguish the muon signal. The produced muons could be slowed down to stop inside the detector. Then generally after an average lifetime $2.2 \mu \mathrm{s}$ it would decay into an electron and two neutrinos $\left(v_{e}\right.$ and $\left.v_{\mu}\right)$ for lepton conservation. The neutrinos escape from the detector easily, but the electron has an energy varying from 0 to $51 \mathrm{MeV}$ which supply a measurable signal in the detector. The detected time of the electron could be used to estimate the lifetime of muons. In general, the scintillator is a good choice to detect and absorb muons since the muon lifetime $2.2 \mu \mathrm{s}$ is much longer than the typical attenuation time of scintillator. There were lots of cosmic ray experiments in which scintillator detectors were used to determine muon lifetime ${ }^{[1,28]}$. Nevertheless, in those experiments, muons were detected event by event, which were quite different from the case here, where millions of muons are generated simultaneously in one laser shot among a strong radiation background.

The lifetime measurement would give an explicit evidence for muon production by a short pulse laser. Furthermore, because muons decay later than the laser 'shot time' by hundreds of ns, the radiation background would attenuate dramatically. To stop muons of different energy in the scintillator, water with different radiation lengths could be employed.

In this paper, we show results of the Geant4 simulation of muon diagnostics process described above. In Section 2, the Geant4 simulation setup of the detection system was described. In Section 3, the dependence of detection efficiency

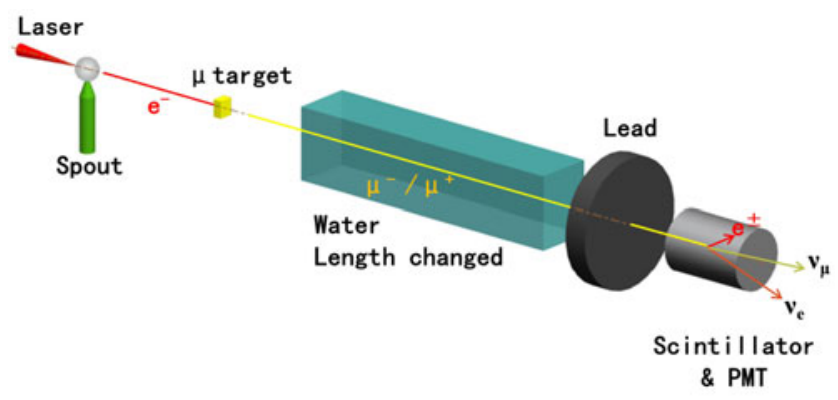

Figure 1. The schematic of dimuon production and diagnostics. Muons were produced through Bethe-Heitler pair production process by photons generated from bremsstrahlung of laser wakefield accelerated electrons in high $\mathrm{Z}$ material (muon target). The produced muons flied along the direction of electron beam into the water to loss energy and stop in the scintillator detector. Lead was used to reduce the radiation background.

on muon energy was derived. In Section 4, the muon lifetime measurements with different yields were discussed. After that the paper ends with a short discussion and a summary.

\section{Simulation setup of the diagnostic system}

The dimuons can be produced by a short pulse laser as shown in Figure 1. As discussed in the Introduction, the 'detection system' consists of three parts: water, lead and the scintillator. Here scintillator is used to catch muons. To stop muons in the detector efficiently, a massive scintillator is needed.

After that an electron with energy range of $0-51 \mathrm{MeV}$ is produced which have a maximum stopping range around $20 \mathrm{~g} / \mathrm{cm}^{2}$. The volume of the scintillator is chosen to be $30 \mathrm{~cm} \times 30 \mathrm{~cm} \times 30 \mathrm{~cm}$. A bigger scintillator would catch the muons more efficiently; however, the collection of scintilla light would be harder.

A layer of $5 \mathrm{~cm}$ lead is introduced to shield the radiation background in the experiment. Since the detection time is later than the laser shot time by several hundreds of ns, only very few secondary particles such as high-energy photons and neutrons have effects on muon detection. It is located behind the water and close to the scintillator. Muon scattering with the lead shield would enlarge the emittance of muon source resulting in lowering the detection efficiency.

Considering the finite volume of the scintillator, only muons with proper energy (less than $100 \mathrm{MeV}$ ) would stop inside the detector and decay consequently. In the case of higher-energy muons, water is employed to decelerate the muons by the minimum ionization process $\left[2 \mathrm{MeV} \cdot\left(\mathrm{g} \cdot \mathrm{cm}^{-2}\right)^{-1}\right.$ in water] while keeping the beam emittance. Water also serves as the radiation shield among the short pulse laser shots. Other materials such as plastic or concrete can also be used here; however, considering the convenience and cost, water is the best choice in our experiment. 
The detection system was simulated by using the Monte Carlo code Geant4. Geant4 is a toolkit for the simulation of the passage of particles through matter ${ }^{[29,30]}$. Its areas of application include high energy, nuclear and accelerator physics. In this paper, the most important thing is to simulate the 'successful catch' of a muon in the detection system. A 'successful catch' means that, after transport through water and lead shields, a muon stops inside the scintillator and decay consequently. After a mean lifetime of $2.2 \mu \mathrm{s}$, the muon decays and an electron is produced, which deposits energy inside the scintillator. The discussion on detection efficiency and muon lifetime measurement given below are all based on the 'successful catch' muon event definition.

\section{Detection efficiency}

To simulate 'successful catch' muon events by Geant 4 , a muon source is placed on the axis of the detection system with a flat energy distribution from 200 to $1000 \mathrm{MeV}$. The laser wakefield accelerated electron beam typically has a radius with $100 \mu \mathrm{m}$, and therefore could be considered as a point source. Muons produced, hence, have a spatial distribution less than several $\mathrm{mm}$ after penetrating the muon target with $\mathrm{cm}$ thickness. Since this is much smaller than the size of the detection system, the muon source is taken as a point source without spatial distribution for convenience.

The physical processes of muons including decay, nuclear reaction, multi-scattering, ionization, bremsstrahlung radiation and electron pair production are considered in the simulation. Information of electrons decayed from muons inside the scintillator is recorded to get a 'successful catch' event.

To 'successfully catch' muons of different energy, the water length is changed from 60 to $380 \mathrm{~cm}$ with a $40 \mathrm{~cm}$ step. The cross-section of water in the simulation is $50 \mathrm{~cm} \times$ $50 \mathrm{~cm}$.

Totally $5 \times 10^{4}$ muons were injected into the detection system. For definite water length of the detection system setting, only a part of muons with suitable energy can be stopped inside the scintillator and decayed correspondingly. Muons with higher or lower energy would depart from the detection system or stop inside the water, which makes it unable to give a measurable scintilla signal. Therefore, every peak of the black line shown in Figure 2(a) denotes the 'successful catch' muons in one water length setting. We do the simulations 9 times for water length settings from 0.6 to $3.8 \mathrm{~m}$; thus, nine peaks appear in the black line in Figure 2(a). Summing over the nine peaks gives the red line.

For finite scintillator volume, the individual peak in Figure 2(a) followed a Gaussian distribution. The $\pm 3 \sigma$ region was taken as the energy range at a definite water length. The detection efficiency of the diagnostics was defined as the ratio of the 'successful catch' muon event number to the total number of muons corresponding to the energy range
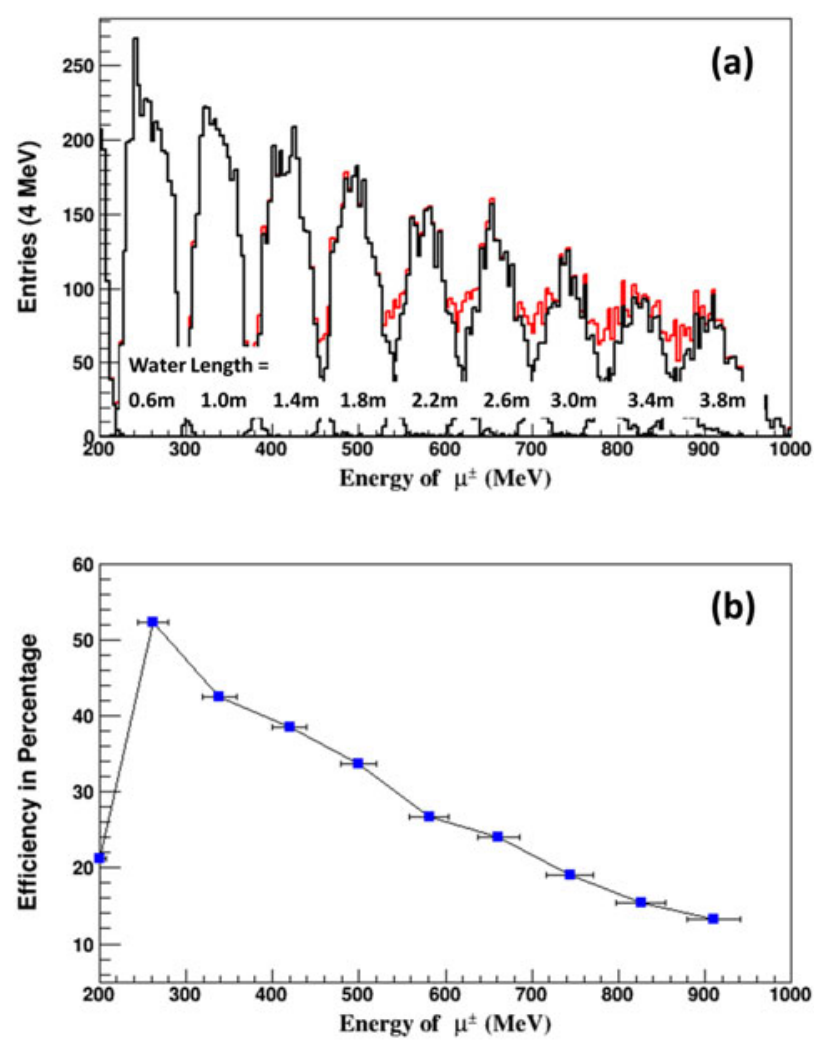

Figure 2. (a) The 'successful catch' muon events as a function of the primary muon energy at different water lengths and (b) detection efficiencies obtained by simulation.

at a definite water length. It is shown in Figure 2 that the detection system can cover an energy range around 120 to $180 \mathrm{MeV}$ ( $\pm 3 \sigma$ region) under different water length settings from 0.6 to $3.8 \mathrm{~m}$ according to the primary energies of muons from 200 to $1000 \mathrm{MeV}$. It is not very surprising as muons with lower energy accept fewer scattering from shorter water length of the detection system. Thereby high detection efficiency up to $52 \%$ could be achieved at a lower primary muon energy while the efficiency decreases to $14 \%$ at a higher primary muon energy. The reason is the longer water length needed to decelerate muons also scatters the muons and therefore decreases the number of muons entering the scintillator.

\section{The muon lifetime diagnostics}

The muon lifetime could be derived by measuring the scintilla light produced by the electrons decayed from muons stopped inside the scintillator detector. When abundant muons are stopped inside the detector, electrons are generated accordingly and deposited rapidly. Thus, in the large sample limit the attenuation of the scintilla light followed an exponential distribution with an average time of the muon lifetime. 


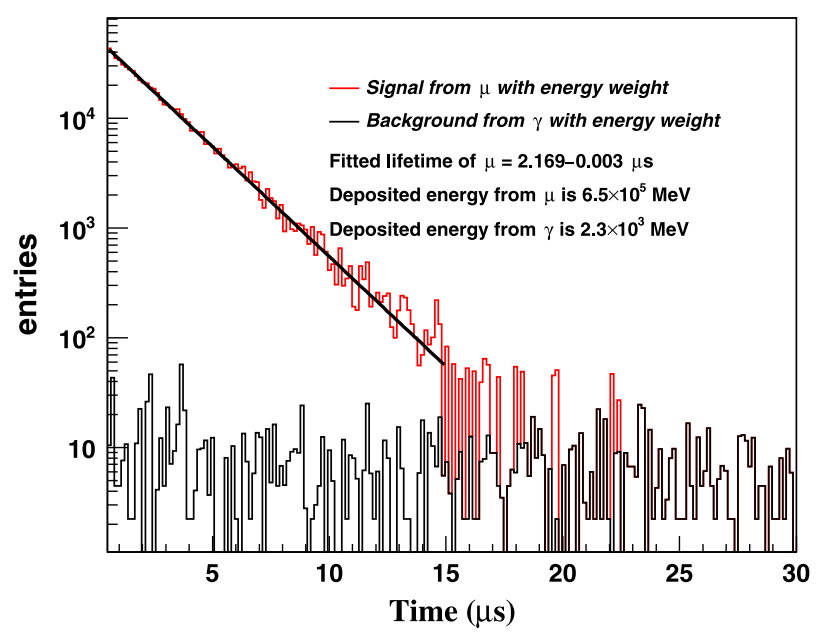

Figure 3. The deposited energy of electrons decayed from 'successful catch' muons as a function of time. The linear background shows radiation contamination from 50,000 photons entering the detection system with a flat energy distribution from 200 to $1000 \mathrm{MeV}$.

In general, the scintilla light yield is proportional to the deposited energy of the electrons but with a coefficient depending on the parameter of definite scintillator. For generalization, only the deposited energy of electrons was considered in this paper.

In the simulation discussed in Section 2, the deposited energy of electrons from the 'successful catch' muons as a function of the time was shown in Figure 3. It is approximately an exponentially decaying distribution presented. The linear background is due to a sample of 50,000 photons that enter the detection system with a flat energy distribution from 200 to $1000 \mathrm{MeV}$. Almost all radiation background of photons were generated within hundreds of nanoseconds which is much shorter than the muon lifetime; therefore could be neglected in the measurement.

It is worth mentioning that the discussion above was obtained in a large sample limit assumption. For a small quantity of muons, since the electrons had an energy range from 0 to $51 \mathrm{MeV}$, the fluctuation in the tail of the distribution would be enhanced. Furthermore, in cases when only a few muons generated, the detection system can be run at muon counting mode, in which muon events could be identified as isolated peaks in the equipment, such as an oscillograph. The lifetime of muons can still be derived with the maximum likelihood fit on those isolated peaks.

\section{Summary}

Muons produced by short pulse lasers can serve as a new type of muon source that have potential advantages of high intensity, small source emittance, short pulse duration and low cost. The first step before we can apply it to fundamental problems of elementary physics such as neutrino physics, lepton physics or be used in $\mu \mathrm{SR}$, muon radiography, is to validate the production in experiment. Here we proposed a new detection system to discriminate high flux muon signal from strong radiation background among the short pulse laser shot by measuring the muon lifetime.

The detection system consisted of three parts: water, lead and scintillator. The scintillator detector is used to stop muons and catch the electrons from muon decays. Water and lead are used to decelerate the muons by ionization processes so that the muons would finally stop inside the scintillator. In addition, they also serve as the shielding against strong radiation background.

A Geant 4 simulation of the whole detection system was performed. The 'successful catch' muon events were recorded to determine the detection efficiency of the system. It was shown that an efficiency up to $52 \%$ could be achieved for low-energy muons around $200 \mathrm{MeV}$ but it decreases to $14 \%$ for high-energy muons up to $1000 \mathrm{MeV}$. The reason is that for high-energy muons, a longer water tank is needed to stop it which also increases the emittance of muon beams. By recording the deposited energy inside the scintillator detector, the muon lifetime could be derived from attenuation of the scintilla light of electrons from muon decays. It is also shown that the detection system can distinguish the muon signal from a strong radiation background such as high-energy photons. The detection system can be easily calibrated by the cosmic ray muons. After that the yield and energy spectrum of muons generated by a short pulse laser would be obtained straightforwardly in experiment.

The detection system can also be run at a counting mode in case of low muon flux. Considering the cross-section of the scintillator is $30 \mathrm{~cm} \times 30 \mathrm{~cm}$ and the detection time interval $(\approx 30 \mu \mathrm{s})$, the cosmic ray muon background is less than $4.5 \times$ $10^{-4}$ in one shot by accident. Thus, once a muon signal is observed, it can be identified as a muon produced by a short pulse laser.

The detection system can be taken as a basic method to measure muons produced in laser experiments. For example, with the development of ion acceleration by a short pulse laser, muons generated by proton beams with energy exceeding muon generation threshold seem to be quite possible in the near future.

\section{Acknowledgement}

This work was supported by the Science Challenge Project (No. JCKY2016212A505) and the CAEP Foundation (No. 2014A0102003).

\section{References}

1. V. W. Hughes and C. S. Wu, Muon Physics (Academic Press, 1977).

2. K. Nagamine, Introductory Muon Science (Cambridge University Press, 2003).

3. The $\mu^{+} \mu^{-}$Collider Collaboration, Fermilab Conf 96/092, LBNL-38946 (1996). 
4. D. M. Kaplan, Fermilab-Conf-00/019, LBNL-38946 (2000).

5. S. J. Blundell, Contemp. Phys. 40, 175 (1999).

6. K. N. Borozdin, G. E. Hogan, C. Morris, W. C. Priedhorsky, A. Saunders, L. J. Schultz, and M. E. Teasdale, Nature 422, 277 (2003).

7. H. Yi, Z. Zeng, X. Wang, J. Cheng, and J. Li, High Power Laser Part. Beams 24, 2987 (2012).

8. H. K. M. Tanaka, T. Nakano, S. Takahashi, J. Yoshida, M. Takeo, J. Oikawa, T. Ohminato, Y. Aoki, E. Koyama, H. Tsuji, and K. Niwa, Earth Planet. Sci. Lett. 263, 104 (2007).

9. L. J. Schultz, G. S. Blanpied, K. N. Borozdin, A. M. Fraser, N. W. Hengartner, A. V. Klimenko, C. L. Morris, C. Orum, and M. J. Sossong, IEEE Trans. Image Process. 16, 1985 (2007).

10. A. Carne, S. F. J. Cox, G. H. Eaton, and C. A. Scott, Hyperfine Interact. 65, 1175 (1990).

11. Y. Miyake, K. Nishiyama, N. Kawamura, P. Strasser, S. Makimura, A. Koda, K. Shimonura, H. Fujimori, K. Nakahara, R. Kadono, M. Kato, S. Takeshita, W. Higemoto, K. Ishida, T. Matsuzaki, Y. Matsuda, and K. Nagamine, Nucl. Instrum. Methods Phys. Res. A 600, 22 (2009).

12. A. Rafeal, B. X. Donath, D. Herlach, D. Maden, I. D. Reid, D. Renker, G. Solt, and U. Zimmermann, Hyperfine Interact. 87, 1105 (1994).

13. G. M. Marshall, Z. Phys. C 56, s226 (1992).

14. H. Burkhardt, S. Kelner, and R. Kokoulin, CERN-SL-2002016 (AP) (2002).

15. S. Hayes, R. Imlay, P. M. Joseph, A. S. Keizer, J. Knowles, and P. C. Stein, Phys. Rev. Lett. 22, 1134 (1969).

16. S. Hayes, R. Imlay, P. M. Joseph, A. S. Keizer, J. Knowles, and P. C. Stein, Phys. Rev. Lett. 24, 1369 (1970).

17. W. A. Barletta and A. M. Sessler, AIP Conf. Proc. 352, 31 (1996).

18. C. Muller, C. Deneke, M. Ruf, G. R. Mocken, K. Z. Hatsagorsyan, and C. H. Keitel, Laser Phys. 19, 791 (2009).

19. X. Wang, R. Zgadzja, N. Fazel, Z. Y. Li, X. Zhang, W. Henderson, Y. Y. Chang, R. Korzekwa, H. E. Tsai, C. H. Pai, H. Quevedo, G. Dyer, E. Gaul, M. Martinez, A. C. Bernstein, T. Borger, M. Spinks, M. Donovan, V. Khudik, G. Shvets, T. Ditmire, and M. C. Downer, Nature Commun. 4, 1988 (2013).

20. W. P. Leemans, A. J. Gonsalves, H. S. Mao, K. Nakamura, C. Benedetti, C. B. Schroeder, C. Toth, J. Deniels, D. E. Mittelberger, S. S. Bulanov, J. L. Vay, C. G. R. Geddes, and E. Esarey, Phys. Rev. Lett. 113, 245002 (2014).

21. H. T. Kim, K. H. Pae, H. J. Cha, I. J. Kim, T. J. Yu, J. H. Sung, S. K. Lee, T. M. Jeong, and J. M. Lee, Phys. Rev. Lett. 111, $165002(2013)$.

22. C. E. Clayton, J. E. Ralph, F. Albert, R. A. Fonseca, S. H. Glenzer, C. Joshi, W. Lu, K. A. Marsh, S. F. Martins, W. B. Mori, A. Pak, F. S. Tsung, B. B. Pollck, J. S. Ross, L. O. Silva, and D. H. Froula, Phys. Rev. Lett. 105, 105003 (2010).

23. A. I. Titov, B. Kampfer, and H. Takabe, Phys. Rev. Sp. Topics Acc. Beams 12, 111301 (2009).

24. Z. Wang, C. Yuan, and N. Qi, High Energy Phys. Nucl. Phys. 27, 716 (2003).
25. A. F. Barbosa, R. Machado, A. Vilar, A. Schiappacassa, R. G. Gama, L. M. de Andrade Filho, and H. P. Lima, Jr., AngraNote 007-2009 (2009).

26. D. Alexander, K. M. Pathak, and M. G. Thompson, J. Phys. A (Proc. Phys. Soc.) 2, 578 (1968).

27. P. Baesso, D. Cussans, C. Thomay, J. J. Velthuis, J. Burns, C. Steer, and S. Quillin, J. Instrum. 8, P08006 (2013) doi:10.1088/1748-0221/8/08/P08006.

28. P. Kammel, Muon capture and muon lifetime, arXiv:nucl-ex/ 0304019v2.

29. S. Agostinelli, J. Allison, K. Amako, J. Apostolakis, H. Araujo, P. Arce, M. Asai, D. Axen, S. Banerjee, G. Barrand, F. Behner, L. Bellagamba, J. Boudreau, L. Broglia, A. Brunengo, H. Burkhardt, S. Chauvie, J. Chuma, R. Chytracek, G. Cooperman, G. Cosmo, P. Degtyarenko, A. Dell'Acqua, G. Depaola, D. Dietrich, R. Enami, A. Feliciello, C. Ferguson, H. Fesefeldt, G. Folger, F. Foppiano, A. Forti, S. Garelli, S. Giani, R. Giannitrapani, D. Gibin, J. J. Gomez Cadenas, I. Gonzalez, G. Gracia Abril, G. Greeniaus, W. Greiner, V. Grichine, A. Grossheim, S. Guatelli, P. Gumplinger, R. Hamatsu, K. Hashimoto, H. Hasui, A. Heikkinen, A. Howard, V. Ivanchenko, A. Johnson, F. W. Jones, J. Kallenbach, N. Kanaya, M. Kawabata, Y. Kawabata, M. Kawaguti, S. Kelner, P. Kent, A. Kimura, T. Kodama, R. Kokoulin, M. Kossov, H. Kurashige, E. Lamanna, T. Lampen, V. Lara, V. Lefebure, F. Lei, M. Liendl, W. Lockman, F. Longo, S. Magni, M. Maire, E. Medernach, K. Minamimoto, P. Mora de Freitas, Y. Morita, K. Murakami, M. Nagamatu, R. Nartallo, P. Nieminen, T. Nishimura, K. Ohtsubo, M. Okamura, S. O’Neale, Y. Oohata, K. Paech, J. Perl, A. Pfeiffer, M. G. Pia, F. Ranjard, A. Rybin, S. Sadilov, E. Di Salvo, G. Santin, T. Sasaki, N. Savvas, Y. Sawada, S. Scherer, S. Sei, V. Sirotenko, D. Smith, N. Starkov, H. Stoecker, J. Sulkimo, M. Takahata, S. Tanaka, E. Tcherniaev, E. Safai Tehrani, M. Tropeano, P. Truscott, H. Uno, L. Urban, P. Urban, M. Verderi, A. Walkden, W. Wander, H. Weber, J. P. Wellisch, T. Wenaus, D. C. Williams, D. Wright, T. Yamada, H. Yoshida, and D. Zschiesche, Nucl. Instrum. Methods Phys. Res. A 506, 250 (2003).

30. J. Allison, K. Amako, J. Apostolakis, H. Araujo, P. Arce Dubois, M. Asai, G. Barrand, R. Capra, S. Chauvie, R. Chytracek, G. A. P. Cirrone, G. Cooperman, G. Cosmo, G. Cuttone, G. G. Daquino, M. Donszelmann, M. Dressel, G. Folger, F. Foppiano, J. Generowicz, V. Grichine, S. Guatelli, P. Gumplinger, A. Heikkinen, I. Hrivnacova, A. Howard, S. Incerti, V. Ivanchenko, T. Johnson, F. Jones, T. Koi, R. Kokoulin, M. Kossov, H. Kurashige, V. Lara, S. Larsson, F. Lei, O. Link, F. Longo, M. Maire, A. Mantero, B. Mascialino, I. McLaren, P. Mendez Lorenzo, K. Minamimoto, K. Murakami, P. Nieminen, L. Pandola, S. Parlati, L. Peralta, J. Perl, A. Pfeiffer, M. G. Pia, A. Ribon, P. Rodrigues, G. Russo, S. Sadilov, G. Santin, T. Sasaki, D. Smith, N. Starkov, S. Tanaka, E. Tcherniaev, B. Tomé, A. Trindade, P. Truscott, L. Urban, M. Verderi, A. Walkden, J. P. Wellisch, D. C. Williams, D. Wright, and H. Yoshida, IEEE Trans. Nucl. Sci. 53, 270 (2006). 